Umgebung einer singulären Stelle oder Linie, Acta Societatis Scientiarum Fennicae, vol. 50 (1922).

6. R. Nevanlinna, Eindeutige analytische Funktionen, Berlin, 1936.

7. E. C. Titchmarsh, On integral functions with real negative zeros, Proc. London Math. Soc. (2) vol. 26 (1927) pp. 185-200.

8. - Theory of functions, 2d ed., Oxford, 1939.

Nagoya Institute of Technology

\title{
CONVERGENCE OF SEQUENCES OF COMPLEX TERMS DEFINED BY ITERATION
}

\section{A. G. AZPEITIA}

In a paper by J. Aczel [1], the following result is established: Let $m\left(x_{1}, x_{2}, \cdots, x_{p}\right)$ be a continuous real function of the real variables $x_{i}\left(i=1,2, \cdots, p,-\infty<a<x_{i}<b<+\infty\right)$ such that: (a) $m(x, x, \cdots, x)=x$ for any $x$ in $(a, b)$, and (b) $m\left(x_{1}, x_{2}, \cdots, x_{p}\right)$ is strictly increasing with respect to all $x_{i}$. Then the sequence $a_{n}$ $=m\left(a_{n-p}, a_{n-p-1}, \cdots, a_{n-1}\right)$ with the initial terms $a_{1}, a_{2}, \cdots, a_{p}$ arbitrarily chosen in $(a, b)$ is convergent.

As corollaries of this theorem, the author proves the theorem of Eneström and Kakeya, ${ }^{1}$ and some well known elementary results. ${ }^{2}$ In fact, the theorem of Eneström and Kakeya is proved to be equivalent to the Aczel result if the function $m$ is of the form

$$
m\left(x_{1}, x_{2}, \cdots, x_{p}\right)=\left(\sum_{i=1}^{p} c_{i} x_{i}\right) /\left(\sum_{i=1}^{p} c_{i}\right), \quad\left(c_{i}>0\right) .
$$

In the present paper, we prove the following more general theorem for the case of complex variables. The nature of the proof is necessarily new because of the impossibility of establishing any relations of inequality for the complex numbers similar to the ones used by Aczel in his proof. The concept of convexity is used to overcome this difficulty and no assumption of analyticity is required.

Received by the editors July 13, 1957 and, in revised form, October 2, 1957 and November 15, 1957.

1 The simplest statement of this theorem is: Let $z$ be any root of $\sum_{j=0}^{m} b_{j} z^{i}=0$ with $b_{m}, b_{m-1}, \cdots, b_{0}$ decreasing positive real numbers. Then, $z$ is of an absolute value less than 1. (See $[2 ; 3 ; 4]$.)

${ }^{2}$ For example, the existence of the limit in the case $m\left(x_{1}, x_{2}\right)=\left(x_{1}+x_{2}\right) / 2$ (see [5]) and consequently in the case of quasi-arithmetic means defined by $m\left(x_{1}, x_{2}\right)$ $=f^{-1}\left\{\left[f\left(x_{1}\right)+f\left(x_{2}\right)\right] / 2\right\},[1$, I. 4$]$. 
THEOREM I. Let $w=f\left(z_{1}, z_{2}, \cdots, z_{p}\right)$ be a complex function of $p$ complex arguments $z_{1}, z_{2}, \cdots, z_{p}$, such that

(1) $f$ is continuous in the set defined by $z_{i} \in D,(i=1,2, \cdots, p)$, where $D$ is a convex region in the complex plane (z),

(2) If $z_{i} \in D$, the point $w=f\left(z_{1}, z_{2}, \cdots, z_{p}\right)$ belongs to the closed convex hull $P$ of the set of points $z_{i}$ and, furthermore, w is different from the extreme points of $P$.

Under the conditions (1) and (2), every sequence $\left\{a_{n}\right\}$ defined by

$$
a_{n}=f\left(a_{n-p}, a_{n-p-1}, \cdots, a_{n-1}\right)
$$

with the initial terms $a_{1}, a_{2}, \cdots, a_{p}$ arbitrarily chosen in $D$, is convergent.

Because of condition (2), the number $w$ is real if $z_{1}, z_{2}, \cdots, z_{p}$ are real numbers, and in particular $\left\{a_{n}\right\}$ is a real sequence if $a_{1}, a_{2}, \cdots, a_{p}$ are real. Consequently, the Theorem I includes as a particular case, the previously mentioned result of $\mathrm{J}$. Aczel, and somewhat improves it, because it is not required that $f\left(z_{1}, z_{2}, \cdots, z_{p}\right)$ is a strictly increasing function.

In general, the assumption (2) cannot be weakened as is easily proved by trivial examples.

The proof of Theorem I will be obtained by using the

THEOREM II. Let $\left\{P_{n}\right\}$ be a sequence of bounded closed convex polygons belonging to $(z)$ with no more than $p$ vertices for every $P_{n}$ and such that $P_{n} \supset P_{n+1}(n=1,2, \cdots)$.

Then, the set $P=\bigcap_{n=1}^{\infty} P_{n}$ is also a bounded closed convex polygon with no more than $p$ vertices.

Proof. We know that $P$ is a bounded closed convex nonempty set (except in the trivial case $P_{n}=\phi$ for some $n$ ), and we begin by establishing the following lemma:

Lemma. Under the conditions of Theorem II, if $s$ is a extreme point of $P=\bigcap_{n=1}^{\infty} P_{n}$, in every neighborhood of $s$ there are infinitely many vertices belonging to the polygons $P_{n}$ (which might coincide totally or partially with s).

Let us suppose that this is not true. Then, for some extreme point $s$ of $P$, there exists a closed circular neighborhood $U$ such that there are, at most, a finite number of vertices in $U$.

Let $\sigma$ be an extreme support of $P$ such that $\sigma \ni s$. Every point of $P$ belongs to one of the two half-planes defined by $\sigma$; for instance, to the half plane containing the point $k$, (see figure), with the convention that $\sigma$ is considered as belonging to such a half plane, which 


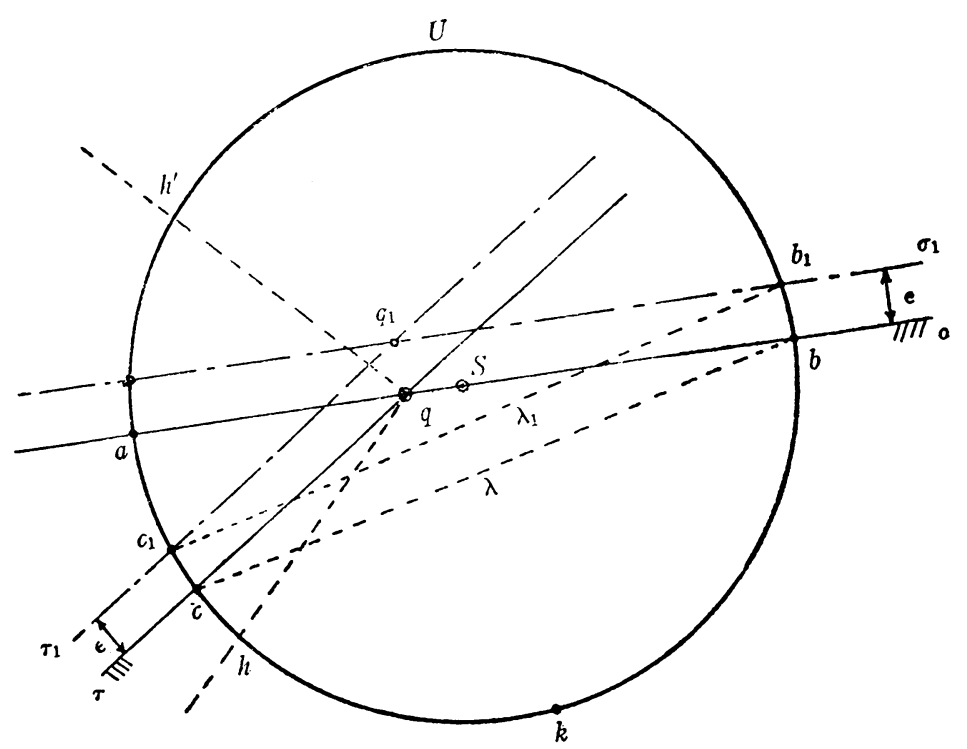

will be denoted by $[\sigma, k]$. And we know also that there are no points of $P$ (different from $s$ ) on one of the two half lines of $\sigma$ with extremity $s$. Let us suppose that this is the case for the half line $[s, a, \infty]$. Consequently, because $P$ is closed and bounded, we can find an angle $\Lambda$ with vertex $q \in(s, a)$ and bisector $[q, a, \infty]$ such that there are no points of $P$ in $\Lambda$. Let $[q, h, \infty]$ and $\left[q, h^{\prime}, \infty\right]$ be the sides of $\Lambda$ (with $h \in[\sigma, k]$ and $h^{\prime} \in[\sigma, k]$ and $h, h^{\prime} \in \gamma \equiv$ boundary of $\left.U\right)$.

If $c$ is an interior point of the arc $(a h)$, the line $\tau$ defined by the points $c$ and $q$ is an extreme support of $P$ and therefore $P \subset[\sigma, k]$ $\cap[\tau, h]$.

Now, we consider two straight lines, $\sigma_{1}, \tau_{1}$, parallel to $\sigma$ and $\tau$ and such that $\sigma_{1} \nsubseteq[\sigma, k], \tau_{1} \nsubseteq[\tau, h]$ and distance $\left(\sigma, \sigma_{1}\right)=\operatorname{distance}\left(\tau, \tau_{1}\right)$ $=\epsilon$. If $\epsilon$ is small enough, the point $s$ is interior to the triangle $b_{1} c_{1} q_{1} \subset U$, because the distances $b b_{1}, c c_{1}, q q_{1}$ are arbitrarily small. Consequently, for some number $n_{0}$, every point of $P_{n}\left(n \geqq n_{0}\right)$ belongs to the convex set $R \equiv\left[\sigma_{1}, k\right] \cap\left[\tau_{1}, h\right]$, and every vertex of $P_{n}$, ( $n \geqq n_{0}$ ) belongs to the convex set $R_{1} \equiv R \cap\left[\lambda_{1}, h\right]$, where $\lambda_{1}$ is the line $b_{1} c_{1}$; and, furthermore, $s \notin R_{1}$.

However, if every vertex of $P_{n},\left(n \geqq n_{0}\right)$ belongs to $R_{1}$, the same is true for every point of $P_{n}$ and $s \notin P=\bigcap_{n=1}^{\infty} P_{n}$, which is contradictory and the lemma is established.

Now, in order to prove Theorem II, it is sufficient to demonstrate that $P$ has no more than $p$ extreme points. 
Let us suppose that this is not true, and let $s_{1}, s_{2}, \cdots, s_{p+1}$ be $p+1$ extreme points of $P$, and $U_{1}, U_{2}, \cdots, U_{p+1}$ a system of closed circular disjoint neighborhoods of $s_{1}, s_{2}, \cdots, s_{p+1}$.

According to the lemma, we can select a partial sequence $\left\{P_{n_{\mu}}\right\}$ from $\left\{P_{n}\right\}$ such that every $P_{n_{\mu}},(\mu=1,2, \cdots)$ has one vertex in $U_{1}$. Obviously, $\bigcap_{\mu=1}^{\infty} P_{n_{\mu}}=P$ and $P_{n_{\mu}} \supset P_{n_{\mu+1}}$ and consequently we can, again, select from $\left\{P_{n_{\mu}}\right\}$ a new sequence $\left\{P_{n_{\mu_{\nu}}}\right\} \subset\left\{P_{n_{\mu}}\right\}$ such that every $P_{n_{\mu_{\nu}}}(\nu=1,2, \cdots)$ has one vertex in $U_{2}$ and also $\bigcap_{\nu=1}^{\infty} P_{n_{\mu_{\nu}}}=P$, $P_{n_{\mu_{\nu}}} \supset P_{n_{\mu_{\nu}+1}}$; etc. After $p+1$ steps, we shall have obtained a partial sequence from $\left\{P_{n}\right\}$ whose polygons have a vertex in every one of the disjoint sets $U_{1}, U_{2}, \cdots, U_{p+1}$. That means that such polygons have at least $p+1$ vertices, which is impossible.

We proceed now to prove Theorem I in the following way:

Let us denote by $P_{n}$ the convex closed hull of the points $a_{n-p}$, $a_{n-p-1}, \cdots, a_{n-1}$. Theorem I will be proved if we show that the polygon, with $p^{\prime} \leqq p$, vertices

$$
P=\bigcap_{n=1}^{\infty} P_{n}
$$

consists of a single point, i.e., if we prove that $p^{\prime}=1$.

Let $h_{1}, h_{2}, \cdots, h_{p^{\prime}}$ be the vertices of $P$. In every circular closed neighborhood of $h_{j},\left(j=1,2, \cdots, p^{\prime}\right)$ there are infinitely many points from the sequence $\left\{a_{n}\right\}$ and we are going to prove that, for every system $S$ of $p^{\prime}$, such neighborhoods $U_{j}$ of $h_{j}$, there exists a number $m_{0}$ such that for $m \geqq m_{0}$, the set of $p$ points $E_{m} \equiv\left(a_{m-p}, a_{m-p-1}, \cdots, a_{m-1}\right)$ has at least one point in every $U_{j}$.

If this is not true, there exists a system $S$ and a partial sequence $\left\{E_{m_{\lambda}}\right\} \subset\left\{E_{m}\right\},(\lambda=1,2, \cdots)$ such that $E_{m_{\lambda}} \cap U_{j}=\phi$ for every $\lambda$ and some $j \leqq p^{\prime}$. Because $j \leqq p^{\prime}$, there will be some $j=j_{0} \leqq p^{\prime}$, such that $E_{m_{\lambda}} \cap U_{j_{0}}=\phi$ for infinitely many $E_{m_{\lambda}}$, which form a new partial sequence $\left\{E_{m_{\lambda_{\mu}}}\right\} \subset\left\{E_{m}\right\}$ with $E_{m_{\lambda_{\mu}}} \cap U_{j_{0}}=\phi,(\mu=1,2, \cdots)$. However, this is impossible because $\bigcap_{\mu=1}^{\infty} P_{m_{\lambda_{\mu}}}=P$ and then we would have $P \cap U_{j_{0}}=\phi$ which is contradictory.

Therefore, there exists a partial sequence of $\left\{a_{n}\right\}$, let us denote it by $\left\{a_{n_{\nu}}\right\}$, such that,

$$
\lim _{\nu=\infty} a_{n_{\nu}}=h_{1}
$$

By the same argument, as we used in Theorem II, we can prove that the sets $E_{n_{\nu}}$ have at least one point in every neighborhood $U_{j}$ of a system $S$ (for every $\nu$ greater than some number $\nu_{0}$ ), and we can 
obtain a new partial sequence $\left\{E_{n_{\nu_{\pi}}}\right\} \subset\left\{E_{n_{\nu}}\right\}$ such that the limits

$$
\lim _{\pi=\infty} a_{n_{\nu_{\pi}}-p}=\alpha_{1}, \lim _{\pi=\infty} a_{n_{\nu_{\pi}}-p-1}=\alpha_{2}, \cdots, \lim _{\pi=\infty} a_{n_{\nu_{\pi}}-1}=\alpha_{p}
$$

exist. Obviously, the set $\left(\alpha_{1}, \alpha_{2}, \cdots, \alpha_{p}\right)$ contains the set $\left(h_{1}, h_{2}, \cdots\right.$, $\left.h_{p}\right)$ and also $\left(\alpha_{1}, \alpha_{2}, \cdots, \alpha_{p}\right) \subset P$.

Now, because $f$ is continuous,

$$
\begin{aligned}
h_{1} & =\lim _{\nu=\infty} a_{n_{\nu}}=\lim _{\pi=\infty} a_{n_{\nu_{\pi}}}=\lim _{\pi=\infty} f\left(a_{n_{\nu_{\pi}}-p}, a_{n_{\nu_{\pi}}} \cdot p-1, \cdots a_{n_{\nu_{\pi}}-1}\right) \\
& =f\left(\alpha_{1}, \alpha_{2}, \cdots, \alpha_{p}\right)
\end{aligned}
$$

and because $P$ is the convex hull of $\alpha_{1}, \alpha_{2}, \cdots, \alpha_{p}$ and $h_{1}$ is a vertex of $P$, the condition (2) implies

$$
h_{1}=h_{2}=\cdots=h_{p^{\prime}} ; \text { i.e. } p^{\prime}=1 \text { and } \lim _{n=\infty} a_{n}=h_{1} .
$$

The author is indebted to Professor David Gale, of Brown University, for his illuminating suggestions concerning the theory of convex sets, and to Professor Allen E. Andersen, of the University of Massachusetts for his help in the final redaction of the manuscript.

\section{REFERENCES}

1. J. Aczel, On some sequences defined by recurrence, Szeged Egyetem Acta Scientiarum Mathematicarum, vol. 13, pp. 136-139.

2. G. Eneström, Härledning af en allmän formel för antalet pensionärer som vid en godtycklig tidpunkt förefinnas inom en sluten pensionskassa, Öfversigt af Kongl. Svenska Vetenskaps-Akademien Förhandlingar vol. 50 (1893) pp. 405-415.

3. - Remarque sur un théorème relatif aux racines de l'équation où tous les coefficients sont réels et positifs, Tôhôku Math. J. vol. 18 (1920) pp. 34-36.

4. S. Kakeya, On the limits of the roots of an algebraic equation with positive coeffcients, Tôhôku Math. J. vol. 2 (1912) pp. 140-142.

5. E. Cesaro and G. Kowalewski, Elementares Lehrbuch der algebraischen Analysis und der Infinitesimalrechnung, Leipzig, 1904, p. 105.

UNIVERSITY OF MASSACHUSETTS 